Journal of Advanced Research in Fluid Mechanics and Thermal Sciences

\title{
A Haar Wavelet Series Solution of Heat Equation with Involution
}

\author{
Burma Saparova ${ }^{1}$, Roza Mamytova ${ }^{1}$, Nurjamal Kurbanbaeva ${ }^{2}$, Anvarjon Akhatjonovich Ahmedov ${ }^{3,}$ \\ Uzgen Institute of Technology and Education, Uzgen, Kyrgyzstan \\ Osh State University, Osh, Kyrgyzstan \\ Pusat Sains Matematik, Universiti Malaysia Pahang, Lebuhraya Tun Razak, 26300 Gambang, Kuantan, Pahang, Malaysia
}

\section{ARTICLE INFO}

\section{Article history:}

Received 2 June 2021

Received in revised form 15 July 2021

Accepted 19 July 2021

Available online 20 August 2021

\section{Keywords:}

Heat equation; Haar wavelet; involution; approximation; numerical analysis

\section{ABSTRACT}

It is well known that the wavelets have widely applied to solve mathematical problems connected with the differential and integral equations. The application of the wavelets possesses several important properties, such as orthogonality, compact support, exact representation of polynomials at certain degree and the ability to represent functions on different levels of resolution. In this paper, new methods based on wavelet expansion are considered to solve problems arising in approximation of the solution of heat equation with involution. We have developed new numerical techniques to solve heat equation with involution and obtained new approximative representation for solution of heat equations.

\section{Introduction}

The wavelets have wide application in engineering sciences and can be applied to solve mathematical problems connected with the differential and integral equations. The application of the wavelets possesses several important properties, such as orthogonality, compact support, exact representation of polynomials at certain degree and the ability to represent functions on different levels of resolution. Haar wavelets are orthonormal wavelets with a compact support. Haar wavelet method is an effective tool for solving ordinary and partial differential equations. There are many researchers who uses Haar wavelets to solve differential and integral equations notably papers [1-3]. A computational method for solving Poisson equations and biharmonic equations which are based on the use of Haar wavelets is investigated in Shi and Cao [4]. The method of two-dimensional Haar wavelets has been applied to obtain the solution of the partial differential equations in Lepik [5]. A collocation method based on Haar wavelet and Kronecker tensor product for solving threedimensional partial differential equations is presented in Lepik [6], where the proposed method is originated from the idea of approximating a sixth-order mixed derivative by a series of Haar wavelet basis functions, which is suitable for numerical solution of all kinds of three-dimensional Poisson and Helmholtz equations.

\footnotetext{
* Corresponding author.

E-mail address: anvarjon@ump.edu.my
}

https://doi.org/10.37934/arfmts.86.2.5055 
In this paper, new methods based on wavelet expansion are considered to approximate the solution of heat equation with involution. The output is a new approximative representation of solution for heat equations.

\section{Haar Wavelets}

There are two functions that play a primary role in wavelet analysis, i.e., the scaling function given by

$\phi(x)=\left\{\begin{array}{c}1, \text { if } 0 \leq x \leq 1 \\ 0, \text { elsewhere }\end{array}\right.$

and the wavelet

$\psi=\left\{\begin{array}{c}1,0 \leq x \leq \frac{1}{2} \\ -1, \frac{1}{2} \leq x \leq 1 \\ 0, \text { otherwise }\end{array}\right.$

These two functions generate a family of functions that can be used to reconstruct signals. Let $M$ be any positive integer. We divide the interval $[0,1]$ into $2 M$ equal subintervals. We intend to do $J$ levels of resolutions, hence we let $2 M=2^{J+1}$.

The Haar wavelet is system of functions defined by the following

$h_{n}=\psi_{j, k}(x)=2^{\frac{j}{2}} \psi\left(2^{j} x-k\right)=\left\{\begin{array}{c}1, \frac{k}{2^{j}} \leq x<\frac{k+0.5}{2^{j}}, \\ -1, \frac{k+0.5}{2^{j}} \leq x \leq \frac{k+1}{2^{j}}, \\ 0, \text { otherwise, }\end{array}\right.$

where $n=2^{j}+k+1, j=0,1,2 \ldots J, k=0,1,2, \ldots 2^{j}-1$. The Haar wavelet system (3) form an orthonormal basis for $L_{2}(\mathbb{R})$. The wavelet expansion of $f \in L_{2}[0,1]$ is given by

$f(x)=\sum_{n=0}^{\infty} a_{n} h_{n}(x)$,

where

$a_{n}=\int_{0}^{1} f(x) h_{n}(x) d x$.

The integration of Haar wavelets is given by

$P_{\alpha, i}(x)=\frac{1}{(\alpha-1) !} \int_{A}^{x}(x-\tau)^{\alpha-1} h_{i}(\tau) d \tau, \alpha=1,2, \ldots n, i=1,2, \ldots, 2 M$

\section{Heat Equation}

Consider a uniform rod of length 1 with non-uniform temperature lying on the $x$-axis from $x=$ 0 to $x=1$. By uniform rod, we mean the density $\rho$, specific heat $c$, thermal conductivity $K_{0}$ are constant. Assume the sides of the rod are insulated and only the ends may be exposed. Let denote 
by $u(t, x)$ a temperature throughout the slice of the rod at the point $x$ and time $t$. Further we assume that the heating process is restricted to the interval $0 \leq t \leq T$, where $T$ is sufficiently big positive number. We consider the second order linear heat equation with involution of the following form

$$
\begin{aligned}
& \frac{\partial u}{\partial t}(T-t, x)=\frac{\partial^{2} u(t, x)}{\partial x^{2}}+f(t, x), 0<t<T, 0<x<1 \\
& u(0, x)=0, x \in[0,1], \\
& u(t, 0)=u(t, 1)=0, t \in[0,1] .
\end{aligned}
$$

To solve the problem (5)-(7), we use the method of approximation by wavelets. Assume that $u(t, x)=v(t) \cdot w(x)$.

We have

$\left.u(t, x)\right|_{t=0}=v(0) \cdot w(x)=0 \Rightarrow v(0)=0$

$\left.u(t, x)\right|_{x=0}=v(t) \cdot w(0)=0 \Rightarrow w(0)=0 ;$

$\left.u(t, x)\right|_{x=1}=v(t) \cdot w(1)=0 \Rightarrow w(1)=0$;

By substituting a function $u(t, x)=v(t) \cdot w(x)$, into the Eq. (5), we obtain

$$
\frac{d v(T-t)}{d t} \cdot w(x)+v(t) \frac{d^{2} w(x)}{d x^{2}}=\lambda v(t) \cdot w(x)
$$

From here

$$
\frac{1}{v(t)} \frac{d v(T-t)}{d t}=\frac{1}{w(x)}\left(\lambda w(x)-\frac{d^{2} w(x)}{d x^{2}}\right) \equiv \mu,
$$

where $\mu$ - certain constant. Thus, if the solution of the problem (5)-(7) is represented as $u(t, x)=$ $v(t) \cdot w(x)$, then the functions $v(t)$ and $w(x)$ are solutions to the following spectral problems:

$$
\frac{d v(T-t)}{d t}=\mu v(t), v(0)=0
$$

and

$$
\frac{d^{2} w(x)}{d x^{2}}=-\gamma w(x), x \in[0,1]
$$


$w(0)=w(1)=0$,

where $\gamma=\mu-\lambda$. The spectral problem

$\frac{d v(T-t)}{d t}=\mu v(t), v(0)=0$,

has an infinite set of eigenvalues

$\mu_{n}=(-1)^{n}\left(n+\frac{1}{2}\right) \frac{\pi}{T}, n=0,1,2, \ldots$,

and their corresponding eigenfunctions

$v_{n}(t)=\sqrt{\frac{2}{T}} \sin \left(n+\frac{1}{2}\right) \frac{\pi t}{T}, n=0,1, \ldots$,

which form an orthonormal basis of the space $L_{2}(0, T)$. We use expansions of the functions $u(t, x)$ and $f(t, x)$ in the system $\left\{\sin \left(n+\frac{1}{2}\right) \frac{\pi t}{T}\right\}_{n=0}^{\infty}$ for fixed $x \in[0,1]$, as follows

$u(t, x)=\sum_{n=1}^{\infty} u_{n}(x) \cdot \sin \left(n+\frac{1}{2}\right) \frac{\pi t}{T}$

$f(t, x)=\sum_{n=1}^{\infty} f_{n}(x) \cdot \sin \left(n+\frac{1}{2}\right) \frac{\pi t}{T}$.

By substitution to the Eq. (5) we obtain

$\sum_{n=1}^{\infty}\left((-1)^{n}\left(n+\frac{1}{2}\right) \frac{\pi}{T} u_{n}(x)-\frac{d^{2} u_{n}(x)}{d x^{2}}\right) \sin \left(n+\frac{1}{2}\right) \frac{\pi t}{T}=\sum_{n=1}^{\infty} f_{n}(x) \sin \left(n+\frac{1}{2}\right) \frac{\pi t}{T}$

By comparing the terms on the left and right sides we obtain

$(-1)^{n}\left(n+\frac{1}{2}\right) \frac{\pi}{T} u_{n}(x)-\frac{d^{2} u_{n}(x)}{d x^{2}}=f_{n}(x)$

$u_{n}(0)=\frac{d u_{n}(0)}{d x}=0$.

We assume that the solution is represented by Haar wavelets (3) as follows

$\frac{d^{2} u_{n}(x)}{d x^{2}}=\sum_{i=1}^{2 M} a_{i} h_{i}(x)$

By integrating the Eq. (11) in the interval $[0, x]$ we have

$$
\begin{aligned}
& \frac{d u_{n}(x)}{d x}=\sum_{i=1}^{2 M} a_{i} P_{i 1}(x)+\frac{d u_{n}(0)}{d x} \\
& u_{n}(x)=\sum_{i=1}^{2 M} a_{i} P_{i 2}(x)+u_{n}(0)
\end{aligned}
$$


where $P_{i 1}(x)$ and $P_{i 2}(x)$ are defined as in Eq. (4). Substitute to ordinary differential equations

$$
(-1)^{n}\left(n+\frac{1}{2}\right) \frac{\pi}{T} \sum_{i=1}^{2 M} a_{i} P_{i 2}(x)-\sum_{i=1}^{2 M} a_{i} P_{i 1}(x)=f_{n}(x)
$$

After we simplify

$\sum_{i=1}^{2 M} a_{i}\left((-1)^{n}\left(n+\frac{1}{2}\right) \frac{\pi}{T} P_{i 2}(x)-P_{i 1}(x)\right)=f_{n}(x)$

We evaluate the latter in the following collocation points $x_{l}=\frac{2 l-1}{4 M}, l=1,2, \ldots, 2 M$.

$\sum_{i=1}^{2 M} a_{i}\left((-1)^{n}\left(n+\frac{1}{2}\right) \frac{\pi}{T} P_{i 2}\left(x_{l}\right)-P_{i 1}\left(x_{l}\right)\right)=f_{n}\left(x_{l}\right)$

The evaluation at collocation points leads to

$\boldsymbol{U}=\boldsymbol{P a}$

$\boldsymbol{U}=\left(u_{n}\left(x_{1}\right), u_{n}\left(x_{2}\right), \ldots, u_{n}\left(x_{l}\right)\right)^{T}$,

$\boldsymbol{a}=\left(a_{1}, a_{2}, \ldots, a_{n}\right)^{T}$,

$\boldsymbol{P}=\left(\begin{array}{ccc}p_{21}\left(x_{1}\right) & \cdots & p_{2 l}\left(x_{1}\right) \\ \vdots & \ddots & \vdots \\ p_{21}\left(x_{l}\right) & \cdots & p_{2 l}\left(x_{l}\right)\end{array}\right)$

By solving the system, we obtain

$\boldsymbol{a}=\boldsymbol{P}^{-1} \boldsymbol{U}$

which substitute into the Eq. (11) yields the following

$\frac{d^{2} u_{n}(x)}{d x^{2}}=\boldsymbol{H} \boldsymbol{P}^{-1} \boldsymbol{U}$

where we denoted

$\boldsymbol{H}=\left(\begin{array}{ccc}h_{1}\left(x_{1}\right) & \cdots & h_{l}\left(x_{1}\right) \\ \vdots & \ddots & \vdots \\ h_{1}\left(x_{l}\right) & \cdots & h_{l}\left(x_{l}\right)\end{array}\right)$

\section{Conclusion}

We have developed new methods based on wavelet expansion are considered to solve problems arising in approximation of the solution of heat equation with involution. New numerical techniques are modified to solve heat equation with involution and obtained new approximative representation for solution of heat equations. 


\section{References}

[1] Coult, Nicholas. "Explicit formulas for wavelet-homogenized coefficients of elliptic operators." Applied and Computational Harmonic Analysis 21, no. 3 (2006): 360-375. https://doi.org/10.1016/i.acha.2006.04.002

[2] Fan, Wei, and Pizhong Qiao. "A 2-D continuous wavelet transform of mode shape data for damage detection of plate structures." International Journal of Solids and Structures 46, no. 25-26 (2009): 4379-4395. https://doi.org/10.1016/j.ijsolstr.2009.08.022

[3] Mrázek, Pavel, and Joachim Weickert. "From two-dimensional nonlinear diffusion to coupled Haar wavelet shrinkage." Journal of Visual Communication and Image Representation 18, no. 2 (2007): 162-175. https://doi.org/10.1016/j.jvcir.2007.01.002

[4] Shi, Zhi, and Yong-yan Cao. "A spectral collocation method based on Haar wavelets for Poisson equations and biharmonic equations." Mathematical and Computer Modelling 54, no. 11-12 (2011): 2858-2868. https://doi.org/10.1016/j.mcm.2011.07.006

[5] Lepik, Ülo. "Application of the Haar wavelet transform to solving integral and differential equations." In Proceedings of the Estonian Academy of Sciences, Physics, Mathematics, vol. 56, no. 1. 2007. https://doi.org/10.1117/12.736416

[6] Lepik, Ulo. "Solving differential and integral equations by the Haar wavelet method; revisited." International Journal of Mathematics \& Computation 1, no. N08 (2008): 43-52.

[7] Lepik, Ülo. "Solving PDEs with the aid of two-dimensional Haar wavelets." Computers \& Mathematics with Applications 61, no. 7 (2011): 1873-1879. https://doi.org/10.1016/i.camwa.2011.02.016

[8] Singh, Inderdeep, and Sheo Kumar. "Wavelet methods for solving three-dimensional partial differential equations." Mathematical Sciences 11, no. 2 (2017): 145-154. https://doi.org/10.1007/s40096-017-0220-6

[9] Lepik, Ülo. "Numerical solution of differential equations using Haar wavelets." Mathematics and Computers in Simulation 68, no. 2 (2005): 127-143. https://doi.org/10.1016/j.matcom.2004.10.005

[10] Aziz, Imran, and Božidar Šarler. "The numerical solution of second-order boundary-value problems by collocation method with the Haar wavelets." Mathematical and Computer Modelling 52, no. 9-10 (2010): 1577-1590. https://doi.org/10.1016/j.mcm.2010.06.023

[11] Aziz, Imran, A. S. Al-Fhaid, and Ajmal Shah. "A numerical assessment of parabolic partial differential equations using Haar and Legendre wavelets." Applied Mathematical Modelling 37, no. 23 (2013): 9455-9481. https://doi.org/10.1016/i.apm.2013.04.014

[12] Aziz, Imran, and Božidar Šarler. "Wavelets collocation methods for the numerical solution of elliptic BV problems." Applied Mathematical Modelling 37, no. 3 (2013): 676-694. https://doi.org/10.1016/i.apm.2012.02.046

[13] Aziz, Imran. "New algorithms for the numerical solution of nonlinear Fredholm and Volterra integral equations using Haar wavelets." Journal of Computational and Applied Mathematics 239 (2013): 333-345. https://doi.org/10.1016/i.cam.2012.08.031

[14] Aziz, Imran, and Muhammad Fayyaz. "A new approach for numerical solution of integro-differential equations via Haar wavelets." International Journal of Computer Mathematics 90, no. 9 (2013): 1971-1989. https://doi.org/10.1080/00207160.2013.770481

[15] Khan, Ansab Azam, Khairy Zaimi, Suliadi Firdaus Sufahani, and Mohammad Ferdows. "MHD Flow and Heat Transfer of Double Stratified Micropolar Fluid over a Vertical Permeable Shrinking/Stretching Sheet with Chemical Reaction and Heat Source." Journal of Advanced Research in Applied Sciences and Engineering Technology 21, no. 1 (2020): 1-14. https://doi.org/10.37934/araset.21.1.114

[16] Halim, Nur Fazlin Che, and Nor Azwadi Che Sidik. "Nanorefrigerants: A Review on Thermophysical Properties and Their Heat Transfer Performance." Journal of Advanced Research in Applied Sciences and Engineering Technology 20, no. 1 (2020): 42-50. https://doi.org/10.37934/araset.20.1.4250 